Fecha de recepción: diciembre 2012 Fecha de aceptación: septiembre 2013 Versión final: septiembre 2014

\section{Hacia un diseño integral}

Luis Rodríguez Morales *

Resumen: En el presente texto se hace una revisión de las posturas habituales en la docencia del diseño y se presenta la estructura que, para las asignaturas de proyectos, se ha adoptado en la Licenciatura en Diseño de la UAM-Cuajimalpa. Se presenta el perfil de egreso, los lineamientos y conceptos básicos de un diseño integral, y los procedimientos para seleccionar las problemáticas que se presentarán a los alumnos. Se describe el proceso general para definir problemas a partir de problemáticas complejas.

Palabras clave: creatividad - diseño integral - perfil de egreso - problemáticas complejas.

[Resúmenes en inglés y portugués en la página 117]

${ }^{(*)}$ Diseñador Industrial por la Universidad Iberoamericana. Doctorado en Teoría e Historia de la Arquitectura por la Universidad Nacional Autónoma de México. Docente-investigador en la Universidad Autónoma Metropolitana Cuajimalpa, Miembro del cuerpo académico de Evaluación del Diseño Centrada en el Usuario. Actualmente Jefe del Departamento de Teoría y Procesos del Diseño.

\title{
Introducción
}

La cuestión de la creatividad es, sin duda alguna, fundamental al proceso de formación de los diseñadores. Para enfrentar esta situación, en ocasiones se busca el apoyo de técnicas diversas que buscan estimular el desarrollo de esta -que a veces parece ser misteriosa-capacidad intelectual. Otra manera de estimular la creatividad es aquella que podemos denominar 'preparar el escenario', es decir establecer una estrategia didáctica que reúna aquellos elementos y que permita el surgimiento de insights o ideas diferentes, que después de un arduo proceso de propuesta-evaluación, nos lleve a configurar una respuesta adecuada.

Otro aspecto importante a considerar es el del problema de diseño en sí mismo. En sistemas tradicionales los profesores ofrecen a los alumnos un brief o definición del problema y el alumno se encarga del análisis del problema como se le propone y ofrece-después de un cierto proceso- una solución. Una postura más crítica, es aquella que establece que los problemas de diseño, en realidad, se construyen. Esta postura tiene su origen en el trabajo de Horst Rittel. 
Rittel propone en su noción de wicked problems ${ }^{1}$ (Protzen y Harris, 2010), que los problemas de diseño en realidad no se pueden definir en su inicio y que la labor del diseñador empieza a partir de la percepción de un desequilibrio en el entorno, y es precisamente a través del esfuerzo por definir objetivos y en consecuencia los criterios de evaluación final, que el diseñador, finalmente, logra establecer algunos parámetros, si bien no llega a desarrollar una definición formal del problema. En consecuencia el inicio de este proceso es dialógico entre el diseñador, la situación descrita (que usualmente llamamos brief), la situación percibida y aquellos involucrados en la situación (usuarios, productores, financieros, distribuidores, etcétera).

Lo anterior implica que, como decíamos líneas arriba, los problemas de diseño se construyen y este es el inicio real del proceso creativo. En la propuesta que aquí se presenta se parte de estas ideas y en consecuencia se describen las condiciones para preparar el escenario en el que los alumnos aplican conocimientos, habilidades y actitudes para llegar a soluciones creativas e innovadoras.

\section{Antecedentes}

La Universidad Autónoma Metropolitana (UAM) se fundó en 1974 con tres unidades o campus y asumió una estructura de Departamentos reunidos en Divisiones para su funcionamiento. En 2005 la Universidad, decidió establecer una cuarta unidad en la zona de Cuajimalpa al poniente de la Ciudad de México. Dentro de los objetivos que se establecieron para esta nueva unidad, está la búsqueda de nuevas estructuras académicas que de alguna manera renueven las existentes, hasta esa fecha, en la UAM.

En las unidades originales (Azcapotzalco y Xochimilco), las carreras relativas al diseño se agruparon en la División de Artes y Ciencias para el Diseño (CyAD), en la que se reúnen carreras como Arquitectura, Diseño Industrial, Diseño Gráfico, Urbanismo, etcétera.

Para la Unidad Cuajimalpa se buscó una conformación diferente con el propósito, entre otros, de estimular la interdisciplina. Así se creó la División de Ciencias de la Comunicación y Diseño, que reúne a tres licenciaturas: Diseño, Ciencias de la Comunicación y Tecnologías de la Información. El hecho de que estos tres campos del conocimiento estén reunidos en una sola División obedece a un enfoque sobre el tipo de problemas a que se enfrentarán los egresados en un futuro y la orientación interdisciplinaria para resolverlos. Para la carrera de Diseño en particular, se buscó una opción diferente. En vez de optar por las carreras tradicionales que surgen de los campos profesionales (industrial, gráfico, etcétera.) se eligió una visión holística, que ahora denominamos Diseño Integral. En un principio se pensó en que el egresado de esta licenciatura abordaría problemas de productos, gráfica y espacios, sin embargo, al revisar tanto la demanda laboral como el currículo de estudios se observó que este perfil sería inadecuado, por lo que se ha propuesto el siguiente: El egresado de la licenciatura en Diseño de la UAM Cuajimalpa está capacitado para construir y gestionar procesos de diseño integral, por medio de la visualización de escenarios y la generación de estrategias y soluciones creativas e innovadoras, desarrolladas desde una postura crítica y con el apoyo de las tecnologías de la información, a problemas 
surgidos de la relación entre el hombre y su entorno, que se caractericen por ser útiles, usables y preferibles, bajo un enfoque social, ambiental y económicamente responsable. Debido a que el perfil de egreso es central al tipo de creatividad que se espera que los diseñadores muestren en su desempeño profesional, en las siguientes líneas se explican los aspectos principales de esta propuesta.

\section{Particularidades del perfil de egreso}

[...] construir y gestionar procesos [...] El concepto de construcción es central al Diseño desde la conformación del Movimiento Moderno. En nuestro caso, se refiere a un proceso que se inicia desde la detección de una problemática y continúa con un análisis que desemboca en un diagnóstico, que a su vez es origen de una labor de indagación y recolección de datos pertinentes que son fundamentales en la creación y desarrollo de ideas o propuestas para resolver la problemática ${ }^{2}$. Este proceso culmina en la especificación de los elementos necesarios para la materialización de la solución propuesta y su evaluación. Por otro lado, el concepto de gestión se refiere a los conocimientos necesarios para "hacer que las cosas sucedan", lo que implica la capacidad de organizar y dirigir el proceso de diseño, considerando todas las variables que intervienen a lo largo del mismo, para lograr la optimización de los recursos disponibles. La gestión implica un alto grado de proactividad, tanto para iniciar como para conducir el proceso. ${ }^{3}$

[...] de visualización de escenarios [...] La noción de construcción de escenarios en diseño se refiere a la capacidad de imaginar una secuencia de eventos o el curso que tomará una acción. En el caso del diseño, un escenario se entiende como el ámbito en que se desarrolla una acción. Por tanto, el egresado de la licenciatura en diseño deberá tener una alta capacidad para imaginar el desempeño de los resultados del proceso de diseño en su contexto de uso, contemplando los efectos para el usuario, el medio ambiente y la cultura. Otra particularidad de los escenarios es que se enfocan hacia lo que está por ocurrir, por lo tanto demanda una actitud prospectiva, desarrollada con base en el análisis de los factores actuales.

[...] generación de estrategias [...] El proceso de diseño persigue, entre otros objetivos, el incremento del valor (en sus múltiples dimensiones de uso, de cambio y de signo) de los objetos diseñados. Para alcanzar este objetivo es necesario generar maneras de enfrentar las problemáticas, que si bien pueden desembocar en métodos y tácticas, parten de visiones estratégicas más generales. La construcción de estrategias se puede entender como la planeación de acciones orientadas a la obtención de objetivos, haciendo uso de los recursos disponibles.

[...] soluciones creativas e innovadoras [...] Es importante distinguir entre soluciones creativas, que son aquellas que ofrecen algún aspecto novedoso, ya sea en el aspecto estético o bien en los factores relacionados con el modo de uso o la aplicación de materiales o tecnología en la producción y la innovación, que la OECD define como La instrumen- 
tación de un nuevo o significativamente mejorado producto (bien o servicio), o proceso, un nuevo método de mercadeo, o un nuevo método organizativo en la práctica de los negocios, organización del espacio de trabajo o relaciones externas (Oslo Manual. The Measurement of Scientific and Technological Activities, 2005:2) por tanto la innovación, si bien se basa en la creatividad, se distingue por su significación y generación de valor, ya sea para el sector del mercado a que se enfoca la solución de diseño, o bien para alguno de los involucrados en su desarrollo, afirmando así la orientación del diseño centrada en el usuario.

[...] desarrollada con el apoyo de las tecnologías de la información [...] La evolución de las tecnologías de la información y la comunicación (TIC) ha impactado el desarrollo profesional del diseño, no solo en cuanto a que son herramientas que vuelven más eficiente el proceso de diseño, sino también en el ámbito productivo (sistemas CAD-CAM), por lo que el egresado de la licenciatura en diseño debe mostrar un alto grado de dominio de las posibilidades que las TIC abren al campo del ejercicio profesional. En este sentido debe contemplarse que más allá del uso de software (que es una demanda laboral importante), el egresado deberá ser capaz de interactuar interdisciplinariamente con profesionistas especialistas en el campo de las TIC.

[...] desde una postura crítica [...] El egresado de la licenciatura en diseño, deberá ser capaz de analizar críticamente su desempeño profesional y el entorno en que se desenvuelve. Si bien el egresado deberá poseer las competencias profesionales que le permitan insertarse en un mercado laboral, no se busca tan solo formar profesionales que reproduzcan las condiciones actuales, sino de ciudadanos capaces de criticar su ámbito de desempeño y la complejidad del mismo, de manera tal que sean capaces de ofrecer nuevas respuestas en un sentido amplio; la crítica misma se ve como un punto de partida hacia la generación de innovaciones que busquen un verdadero desarrollo social incluyente y no seguir tan solo los dictados de modas o procesos irracionales ligados al consumismo.

[...] a problemas surgidos de la relación entre el hombre y su entorno [...] El problema central del diseño se encuentra en la interfase entre una tecnología o situación en el contexto (físico y cultural) y el ser humano. En palabras de Bonsiepe

se debe tomar en cuenta que la interfase no es un objeto, sino un espacio en el que se articula la interacción entre el cuerpo humano, la herramienta (artefacto, entendido como objeto o como artefacto comunicativo) y objeto de la acción. Éste es justamente el dominio irrenunciable del diseño industrial y gráfico (Bonsiepe, 1993, p. 17).

En este concepto de interfase encontramos el espacio de acción del diseñador, en el que se reúne la problemática planteada por el entorno, el ser humano y un objeto que actúa como mediación entre ambos. Para el diseñador integral resulta fundamental entender 
con claridad este concepto, pues por su orientación, el campo problemático de la interfase (entendida como un espacio de articulación) representa su campo de acción.

[...] que se caractericen por ser útiles, usables y preferibles [...] Estas tres dimensiones plantean los centros de atención o ejes problemáticos de la interfase. Lo útil se refiere a la dimensión social de lo diseñado, esto es que los productos del proceso de diseño busquen aportar soluciones a problemáticas complejas de la sociedad tales como la sustentabilidad, la inclusión y el desarrollo económico y no centrarse exclusivamente en el perfeccionamiento de objetos aislados de su entorno social, político, económico, cultural y ambiental. Lo usable se refiere a la dimensión personal entendida como las condicionantes ergonómicas, culturales y sociales de las personas que desempeñarán una acción con lo diseñado, ya sea un proceso comunicativo o de desempeño ergonómico; esta dimensión considera los conocimientos de índole cognitiva y física de la ergonomía. Lo preferible se refiere aun ámbito que abarca, por un lado, lo atrayente de un diseño, es decir aquellas características formales que hacen que el objeto resulte atractivo a un grupo de personas o núcleo social y por otro lado, que en cuanto a su costo y precio se encuentre dentro de las posibilidades de adquisición reales de dicho grupo. Estos dos polos se complementan con aquellos factores que hacen que un producto sea elegido con respecto a otro, comprendiendo la compleja relación entre lo agradable y lo económicamente factible, es decir las dimensiones económica y cultural. (Buchanan, 2001).

Este perfil de egreso se inserta dentro del modelo educativo adoptado por la Unidad Cuajimalpa (Fresán y Outón, 2006) y la estructura del plan de estudios, que se compone de cuatro áreas interrelacionadas entre sí: Historia; Teoría y Métodos; Lenguaje, Representación y Comunicación; Procesos y Tecnologías para la producción y el eje medular que los constituyen los Laboratorios de Diseño, que son las asignaturas donde se desarrollan los proyectos de diseño. Las reflexiones y propuestas que se presentan a continuación están enfocadas precisamente a los Laboratorios de Diseño.

\section{Los laboratorios de diseño y sus premisas}

Orientar los cursos de los laboratorios implica a su vez dar coherencia, de manera sistémica, tanto al enfoque como a los contenidos de las otras asignaturas del plan de estudios, debido a que los laboratorios son el eje medular de la licenciatura en diseño, por lo que para alcanzar los objetivos que se proponen en este bloque de asignaturas, es necesario considerar el apoyo y concurrencia de otras habilidades y conocimientos, desarrolladas y adquiridos en otras asignaturas del currículo de estudios.

Para establecer la orientación de estos cursos es necesario, en primera instancia, establecer algunos puntos de partida o premisas que se usarán a lo largo del currículo.

Diseño como generador de valor. Se considera que el objetivo final del proceso de diseño es la generación de algún tipo de valor para alguien. Así, por ejemplo una persona decide 
comprar un cierto objeto, porque este le ofrece algo que aprecia o valora, como puede ser un menor precio, o bien la posibilidad de desempeñar mejor una acción, o tal vez se valoran las características formales o estéticas. Esto implica que hay distintos tipos de valores que un producto ofrece a diversos consumidores o personas involucradas de alguna manera en la cadena financiamiento-producción-distribución-consumo-desecho. A grandes rasgos, los valores que con mayor frecuencia se obtienen de un proceso de diseño son (Baudrillard, 2000): valor de cambio (se refiere a las cuestiones económicas), valor de uso (se refiere al desempeño de un trabajo o aspectos funcionales) y valor de signo (se refiere a las características estéticas). 4

Diseño centrado en el usuario. Si bien es cierto que tradicionalmente el diseño se ha pensado para satisfacer las necesidades del usuario, en este caso el concepto de diseño centrado en el usuario se refiere a los métodos de análisis (algunos de ellos de carácter etnográfico) que se orientan no tan sólo a los aspectos fisiológicos, sino también a los culturales (Press, 2005). Por otro lado, el concepto tradicional de usuario, entendido como una sola persona que utiliza algún artefacto, se ve substituido por el más amplio de stakeholders, término de difícil traducción al castellano y que engloba a todos aquellos que de alguna manera (ya sea física o no) se relacionan con el artefacto (Krippendorff, 2006). Este concepto incluye a las empresas, que en cierta medida también 'usan' los productos de diseño para estimular su desarrollo o, por ejemplo, entidades políticas que "usan" al diseño para comunicarse con amplios sectores de la población. Es en este sentido amplio que usamos el concepto de usuario.

Proceso interdisciplinario. En nuestro caso, la interdisciplina es connatural para la solución de problemas complejos. Debido a que los problemas son diferentes entre sí,es imposible determinar de antemano que otras disciplinas pueden coadyuvar a la solución, por lo que uno de los aspectos que el diseñador integral debe enfrentar es el de definir el tipo de colaboración que debe tener para gestionar un proceso de diseño adecuado a la problemática.

Problemáticas y problemas. La problemática es una categoría más amplia, dentro de la cual el problema es un aspecto delimitado. En nuestra visión del proceso consideramos que el diseñador integral debe ser capaz de enfrentarse a problemáticas, de las cuales se desprenderán problemas específicos.

A partir del marco general establecido en la UAM Cuajimalpa, el perfil de egreso y las premisas mencionadas, podemos decir, a grandes rasgos, que las capacidades que se espera que un diseñador integral muestre en su desempeño, son las siguientes:

- Detectar una problemática

- Identificar y delimitar un problema dentro de la problemática

- Acotar el problema

- Establecer los aspectos relevantes para la solución del problema

- Establecer los objetivos en términos de incremento de algún valor

- Determinar los criterios de evaluación 
- Definir las fuentes de información pertinentes

- Generar la estrategia adecuada

- Gestionar el proceso de diseño

- Comunicar el proceso de diseño y sus resultados a distintas audiencias

- Evaluar la totalidad del proceso y no sólo los resultados u artefactos diseñados

\section{Los laboratorios de diseño y las problemáticas}

A partir de los conceptos enumerados líneas arriba, resulta evidente que la selección de la problemática es central para alcanzar los objetivos planteados. La orientación de la licenciatura en diseño de la UAM-Cuajimalpa es la de un diseñador integral, que es una categoría que si bien guarda cierta relación con los campos profesionales tradicionales (industrial, gráfico, digital, etc.), difiere de ellos y por lo tanto, en cierta medida, aún es difícil apuntar con exactitud el tipo de problemas que resolverán nuestros egresados y bajo que enfoque lo harán. Por lo tanto, la habilidad de encontrar, identificar y acotar un problema será una de las más importantes para nuestros egresados; estos deben tener la capacidad para enfrentarse a distintas situaciones y dar respuesta, en principio, a esta pregunta: ¿qué puede hacer un diseñador integral ante una cierta situación? Es por esto que se propone el estudio de problemáticas y no de temas específicos.

La actitud tradicional de los diseñadores es esperar que 'el cliente' pida la solución a un problema definido. Por ejemplo un diseñador industrial esperaría que ante una problemática como puede ser la educación, se le pida desarrollar algún objeto o equipamiento, mientras que un gráfico esperaría que le pidan desarrollar algún material didáctico (impreso o digital) y así para las distintas especialidades profesionales del diseño. Para el caso del diseñador integral, esperamos que sea capaz de identificar y definir un problema dentro de la problemática general. Como se mencionó líneas arriba, deberá plantearse en primera instancia preguntas tales como ¿qué es educar en este contexto específico? ¿cuántas maneras hay de educar? ¿cuál es el propósito de educar? y después ¿qué puede hacer un diseñador integral ante esta situación?

Así, es de esperar que un diseñador integral, ante las primeras preguntas sea capaz de plantear y analizar las múltiples dimensiones de la educación: por ejemplo dentro de un sistema escolarizado, o también educar en un sentido más amplio como puede ser dar elementos de educación vial a todos los habitantes de la ciudad, o proporcionar elementos de educación en salud a un sector específico de la población. Aunado a esta reflexiones, deberá tener la capacidad de investigar en busca de datos específicos (datos "duros") que den sustento a sus reflexiones y con los cuales podrá argumentar sobre la pertinencia de su propuesta.

A partir de la detección de las múltiples opciones que presenta una problemática, entonces el diseñador integral deberá ser capaz de proponer un problema específico, al tiempo que ha detectado los distintos conocimientos, provenientes de otras disciplinas, que deberá articular en el desarrollo del proceso de diseño. Con estos elementos, deberá ser capaz de trazar una estrategia viable, considerando aspectos como recursos disponibles, tiempo, etcétera. Lo mismo podemos decir sobre otros casos. Por ejemplo ante la problemática de salud, 
un diseñador integral, al entrar a una clínica, debería ser capaz de detectar problemas específicos y proponer soluciones que pueden ir desde una señalética apropiada, al mobiliario o el diseño de los cubículos o consultorios, o una propuesta para una campaña para un fin específico. Es importante hacer notar que estas propuestas se entienden como una interfase y no solamente en el sentido tradicional que damos a estos artefactos. Con esto queremos enfatizar el hecho de que el diseñador debe ser capaz de entender que el producto a diseñar debe ser tal que permita al ser humano relacionarse con su contexto. Es en este sentido que entendemos, de nuevo a manera de ejemplo, que una señalética en un edificio es la interfase que permite al usuario utilizar de manera eficiente un ámbito o espacio, o bien que el propósito del mobiliario no es solamente dar comodidad, sino permitir que un médico interactúe con un paciente de una manera especial. Un aspecto constante a considerar es que estas propuestas deben ofrecer algún incremento de valor a alguien, como ya se ha mencionado

Para armonizar y dar cierta orientación a los cursos, es necesario pensar en una estructura general que nos permita orientar el tipo de problemáticas a que se enfrentarán los alumnos, para lo cual,podemos distinguir ciertas problemáticas comunes a los seres humanos. Debido a que la estructura docente de la UAM se desarrolla con base en trimestres ${ }^{5}$, nuestra propuesta es que estas problemáticas se trabajen a partir del VI trimestre (que equivale a la mitad de los estudios completos, una vez que los estudiantes han recibido los cursos básicos), por lo que los alumnos trabajarían alrededor de una de ellas durante tres trimestres, mientras que en proyecto terminal (que se desarrolla a lo largo de tres trimestres o un año lectivo) se trabaja otra de las problemáticas. Esto implica que habrá varios grupos a la vez trabajando diversos problemas dentro de la misma problemática, con lo que se genera un intercambio entre ellos, lo que enriquece tanto el proceso (particularmente en las fases analíticas y de investigación) para obtener los datos necesarios para acotar los problemas. Los contenidos de los trimestres I al V, se desarrollan conforme a una secuencia que tiene por objetivo proveer a los estudiantes de las herramientas suficientes para enfrentarse a la configuración formal. Esto incluye en los primeros trimestres (I a III) ejercicios fundamentales de composición y geometría. Del tercer trimestre al quinto, los alumnos desarrollan los primeros proyectos de baja complejidad, con el objetivo de estructurar un proceso de diseño. La orientación en estos cursos es, a grandes rasgos, la de realizar ejercicios y no proyectos completos, para que los alumnos puedan desarrollar las habilidades necesarias para el desarrollo de problemas más complejos en los cursos superiores. En los primeros trimestres se espera que los alumnos lleven a cabo varios ejercicios durante el curso, en oposición al desarrollo de un solo proyecto, como se hace del VI trimestre en adelante.

Cabe mencionar que durante los primeros tres trimestres los ejercicios se desarrollan individualmente y a partir del IV trimestre el trabajo se hace en equipos de tres o cuatro alumnos. 


\section{Preferible, usable, útil}

Las categorías de útil, usable y preferible forman parte de los criterios de evaluación (por parte de los profesores) y de autoevaluación de los alumnos con respecto a su trabajo. Estas son categorías de análisis y a la vez características de las soluciones de diseño.

A grandes rasgos, lo preferible se centra en el análisis de los factores (cuantitativos y cualitativos) que hacen que un producto de diseño sea preferido (o deseado) con relación a otro. Para la solución de esta dimensión se puede recurrir a conocimientos sobre retórica visual, composición formal, etc. Si bien el factor "estético" juega un papel importante en esta categoría, no es el único a considerar; también aspectos como el grado de innovación y lo multisensorial, tienen un rol destacado.

Lo usable se refiere a aspectos que, en primera instancia, se refieren a conocimientos sobre ergonomía, tanto física como cognitiva. La usabilidad no sólo se aplica a temas relacionados con interfases digitales, también se apoya en conocimientos sobre tipografía y confort. Esta categoría guarda una fuerte relación con el tradicional concepto de función.

Lo útil se refiere específicamente al contexto social y económico (vale la pena mencionar que lo cultural se encuentra presente en las tres categorías, así como algunos aspectos económicos), y por lo tanto establece una fuerte relación con la viabilidad de lo diseñado y por lo tanto con aspectos empresariales y de realización.

Se espera que estas tres categorías estén presentes en los resultados de los cursos y especialmente en los proyectos terminales se deberán ofrecer argumentos al respecto en los proyectos de los alumnos, sin embargo para el análisis y dominio de ellas, es necesario dosificar su aplicación a lo largo de la carrera.

\section{Los laboratorios de diseño y los énfasis}

En el contexto de los laboratorios de diseño, el énfasis se refiere al grado de intervención directa del profesor en el desarrollo de los proyectos. Así, en los primeros proyectos,se espera que el alumno se inicie en el análisis de la problemática, pero el profesor deberá intervenir y colaborar en la definición del problema. En estos trimestres, debido a que el énfasis se ubica en lo preferible, se pueden plantear más bien ejercicios (y no proyectos completos), de manera que el alumno adquiera confianza en sí mismo y ejercite aspectos como la creatividad (eventualmente también la innovación), por lo tanto, en estos trimestres se pueden realizar varios ejercicios, cada uno de ellos con objetivos claros y evaluables. En trimestres intermedios, se marca un énfasis en lo usable, por lo que la categoría de lo preferible se deja para que el alumno la resuelva, mientras que el profesor colabora de manera directa en el análisis y solución de lo usable. En estos cursos es posible pensar que los alumnos resuelvan dos proyectos por trimestre. Estos serán proyectos de complejidad relativamente baja, recordando que el objetivo del curso no es que el alumno entregue proyectos, sino que aprenda y ejercite la manera de solucionarlos. A diferencia de los trimestres anteriores, en estos se desarrollan proyectos de mediana complejidad y no solamente ejercicios. 
Por último, en el proyecto terminal, los alumnos se hacen cargo de las dos primeras categorías y el profesor colabora en el análisis y solución de lo útil. Es claro que en estos proyectos, los alumnos deben ser más independientes, sin embargo es necesario recordar que los profesores deben enseñar ciertos contenidos que apoyen el desarrollo de la solución. Debido a la complejidad de estos proyectos, los alumnos realizarán tan solo uno, pero a juicio del profesor se podrán desarrollar otros ejercicios breves, que ayuden al desarrollo y consolidación de distintas habilidades y/o conocimientos.

\section{Flujo}

Si bien es cierto que existen múltiples modelos metodológicos para abordar los problemas de diseño, se considera necesario que todos los profesores adopten un modelo de flujo, en el que se indican las fases generales que se deben observar en todos los trabajos. Este modelo de flujo NO pretende substituir a los métodos proyectuales, la aplicación de estos deberá ser uno de los aportes que, en cuanto a contenidos, deben observarse para cada curso y serán parte de los programas específicos que cada uno de los profesores elabora. La ventaja de adoptar un modelo de flujo es que se presentará a los alumnos, de manera reiterada, una visión ordenada que todoel profesorado comparte sobre cómo iniciar el abordaje de un problema. Por otro lado permite desarrollar un lenguaje común que será un primer elemento para coordinar y consolidar los criterios de evaluación.

\section{Las etapas de este flujo son:}

Análisis de la problemática. Implica detectar a los distintos usuarios, sus características generales y sus necesidades, así como el contexto de uso.

Definición del problema. Implica establecer la orientación de la solución y los alcances del proyecto.

Desarrollo de alternativas. Implica la generación de diversas soluciones posibles, atendiendo a los distintos aspectos del problema.

Evaluación. Implica por un lado la selección de la alternativa más viable o que mejor resuelva los requerimientos del problema y por otro, el proceso que permite constatar que el resultado ha cumplido y en que medida con los objetivos iniciales. Este aspecto involucra conocimientos de argumentación y para el análisis de la propuesta en el contexto de la problemática inicial. 
Intencionalmente se ha decidido mantener este flujo en un mínimo de etapas, para permitir la flexibilidad necesaria para que los profesores presenten métodos específicos de proyectación.

$\mathrm{Al}$ igual que en el caso de las categorías generales (preferible, usable y útil), en el caso de las etapas del flujo, también debe haber un énfasis dependiendo del nivel de los cursos. Así, en los primeros trimestres el profesor ayudará a los alumnos en el análisis de la problemática y su evaluación y en menor medida en la fase de definición del problema, mientras que los alumnos deberán aportar más en cuanto al desarrollo de alternativas.

En trimestres intermedios, los profesores aportarán en las fases de análisis y evaluación de la problemática y los alumnos desarrollarán de manera clara las de definición del problema y desarrollo de alternativas. En los proyectos terminales, los profesores se convierten en asesores y se espera que los alumnos se hagan cargo de la totalidad del flujo, además de elegir un método proyectual específico, estimulando de esta manera que los alumnos sean co-responsables de su formación.

\section{Conclusiones generales}

La formación de un diseñador integral es una tarea que apenas se inicia. Cuando partimos de las experiencias acumuladas en generaciones de egresados (como es el caso de las carreras en diseño tradicionales), se requiere de analizar los resultados obtenidos y con base en ellos proponer modificaciones al currículo de estudios. En nuestro caso, debido al actual nivel de experiencias acumuladas, se requiere más bien realizar un ejercicio de prospectiva. A la fecha hay ya tres generaciones de egresados, pero ninguna que haya cursado la licenciatura bajo el sistema descrito en el presente texto, que apenas se empieza a instrumentar. De los cursos que se han impartido con este enfoque, los resultados son promisorios. Es posible detectar en los alumnos una mayor capacidad para identificar problemas dentro de una problemática. Así mismo se observa en los proyectos una mejor integración y desarrollo del concepto de interfase, así como una mayor comprensión de la relación entre los artefactos y el contexto.

Una debilidad en el enfoque del diseño integral, sin duda, es el aspecto relacionado con la producción pues resulta demasiado complejo abordar tecnologías que van desde la impresión de gráficos hasta la materialización de productos. Sin duda es una debilidad, pero es necesario recordar que para nuestra propuesta el eje central es el de la interfase. Es en este aspecto donde nuestros egresados pueden aportar al desarrollo de un país y a la solución de las necesidades. 


\section{Notas}

1. El concepto wicked problems se puede traducir -literalmente- como 'problemas perversos'.

2. Es importante subrayar que en esta idea subyace la noción de que los problemas de diseño se construyen, que es diferente a la postura tradicional en la que los problemas de diseño se "definen" por medio de un brief que el cliente entrega al diseñador.

3. La proactividad es un elemento central en la formación del diseñador integral. Mientras que en un ambiente tradicional el diseñador espera que "el cliente" le presente un problema, en nuestro caso se espera que, frente a una situación dada, el diseñador se pregunte ¿qué puedo yo hacer? y que a partir de esta pregunta construya el problema y el proceso para resolverlo.

4. Estos valores guardan una estrecha relación con las categorías mencionadas líneas arriba con respecto a los productos de diseño: útil, usable, preferible.

5. La totalidad de la licenciatura en diseño se cursa en doce trimestres, equivalente a cuatro años.

\section{Bibliografía}

Baudrillard, J. (2000). El sistema de los objetos. México: Editorial Siglo XXI.

Bonsiepe, G. (1993). Las siete columnas del Diseño. México: UAM-A.

Buchanan, R. (2001). Design Research and the New Learning. En Design Issues. Vol. 17, No 4 pp. 3-23.

Fresán, M. y Outón, M. (2006). Reflexiones sobre el Modelo Educativo de la UAM Cuajimalpa. México: UAM.

Krippendorff, K. (2006). The Semantic Turn: a new foundation for Design. Florida Taylor \& Francis.

Oslo Manual. The Measurement of Scientific and Technological Activities. (2005). Organization for Economic Co-operation and Development. OECD.

Protzen, J.-P. y Harris, D. (2010). The Universe of Design. Horst Rittel's Theories of Design and Planning. Nueva York: Routledge.

Press, M. (2005). The Design Experience: The role of design and designers in the twenty-first century. Londres: Ashgate Publishing Ltd. 
Resumo: Este artigo faz uma revisão das posturas habituais na docência do design e se apresenta a estrutura que, para as matérias de projetos, se adotou na licenciatura em Design da UAM-Cuajimalpa. Apresenta-se o perfil de egresso, os lineamentos e conceitos básicos de um design integral, e os procedimentos para selecionar as problemáticas que se apresentarão aos alunos. Descreve-se o processo geral para definir problemas a partir de problemáticas complexas.

Palavras chave: criatividade - design integral - perfil de egresso - problemática.

Summary: This paper proposes a review of common positions in design teaching and also presents the structure that has been adopted for those subjects of projects in the Bachelor of Design from the UAM-Cuajimalpa. In the article, the outcome profile, the guidelines and basic concepts of a comprehensive design and procedures for selecting the issues to be presented to students are described. It explains the general process to define problems from complex problems.

Keywords: creativity - integral design - issues - outcome profile. 